\title{
Effects of PPARy agonist on the polarization of microglia around hematoma after intracerebral hemorrhage in male rats
}

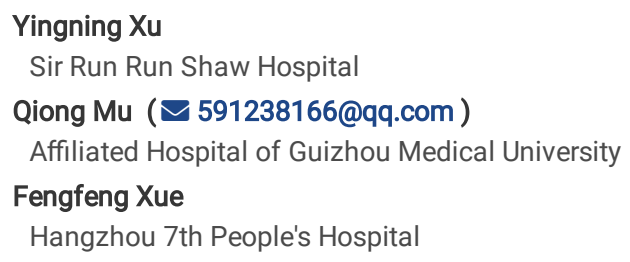




\section{Abstract}

Objective: To study the effect of PPARy agonist (rosiglitazone) on the polarization of microglia around the hematoma after intracerebral hemorrhage in male SD rats.

Materials and Methods: A total of 48 male SD rats were divided into a normal group (Control), the intracerebral hemorrhage model group (ICH), the sodium chloride intervention group ( $\mathrm{NaCl}$ ), and the rosiglitazone intervention group (RSG).ICH model of male rats was established by autologous blood. The neurological function of all groups of male rats was scored at 6, 24, 48, and 72h after modeling. The expression of CD16 (M1 marker) and CD206 (M2 marker) in the peripheral tissues of hematoma was detected by immunohistochemistry and Western blot.

Results: Longa scores of the ICH and RSG group were significantly higher than those of the Control group and $\mathrm{NaCl}$ group at each time (P< 0.05$)$. The RSG group's Longa score at 6,24 , and $48 \mathrm{~h}$ was lower than that of the ICH group $(\mathrm{P}<0.05)$. In the ICH group and RSG group, CD16 reached its peak at $6 \mathrm{~h}$ and then decreased; the expression level of CD16 in the RSG group was lower than that in the ICH group at $6 \mathrm{~h}(\mathrm{P}<0.05)$. The CD206 reached a peak at $24 \mathrm{~h}$; however, the expression level of CD206 in the RSG group was higher than in the ICH group $(P<0.05)$.

Conclusion: PPARy agonist stimulates the activation of M2 microglia , enhancing the ability to remove hematoma microglial cells.

\section{Introduction}

After intracerebral hemorrhage, the blood components such as thrombin, heme, ferrous ions, and other components stimulate the rapid activation of resting microglia, which then differentiate into classic activated state M1 type and alternatively activated state M2 type ${ }^{[1]}$. Activation of M1 microglia-mediated inflammatory cascade reaction releases TNF a, IL - $\beta$, IL - 12, IL - 1 a, IL - 6, IL - 23, CX - CL2 chemokines, REDOX molecular (phagocyte NADPH oxidase, iNOS), causing cytotoxicity, oxidative stress, and inflammation reaction, and eventually damaging cells. On the other hand, the activation of M2-type microglia has anti-inflammatory and neuroprotective effects ${ }^{[2 ; 3]}$. Promoting the transformation of microglia phenotype from M1-type to M2-type has been suggested as a potential treatment for secondary brain damage.

PPAR y reduces axonal injury and its surrounding inflammatory response by activating microglial cells in traumatic brain injury ${ }^{[4]}$. Rosiglitazone (RSG) is one of the most potent agonists of PPAR $y$. RSG can effectively reduce glutamate levels and blood-brain barrier permeability and improve nerve function by upregulating PPAR y expression ${ }^{[5,6]}$, which then further increases CD36 expression. This, in turn, promotes microglia to engulf dead cells and other debris ${ }^{[7]}$, thus achieving cerebral protection by removing lysed red blood cells and toxic substances from the hematoma.

In this study, we examined the effect of PPARy agonist (rosiglitazone) on the polarization of microglia (M1 and M2) around the hematoma after intracerebral hemorrhage in male SD rats.

\section{Materials And Methods}

\section{Reagents}

CD16 rabbit polyclonal antibodies were purchased from Beijing Boorson Biotechnology Co., LTD; CD206, mouse monoclonal antibody, was bought from Proteitech, USA; Goat anti-rabbit/rat working fluid, normal goat serum, and concentrated DAB kit were purchased from Beijing Zhongshan Jinqiao Biology Co., LTD.

\section{Animals and modeling}

Sixty-nine healthy SD male rats, SPF grade, weighing about 250-300g/animal, were provided by the Animal Experiment Center of Guizhou Medical University. All the animals were housed in an environment with a temperature of $22 \pm 1{ }^{\circ} \mathrm{C}$, relative humidity of $50 \pm 1 \%$, and a light/dark cycle of $12 / 12 \mathrm{hr}$. All animal studies (including the mice euthanasia procedure) were done in compliance with the regulations and guidelines of Guizhou Medical University institutional animal care and conducted according to the AAALAC and the IACUC guidelines (Certificate number: SYXK (Guizhou) 2018-0001, Ethics Certificate Number: L1900541).

From 69 rats, 21 rats died (2 during the process of breeding, 2 after the injection of anesthetic, 9 during modeling, 2 during rosiglitazone lavage, and 6 when giving rosiglitazone lavage). Finally, 48 rats were randomly divided into four groups (12 rats in each group): including the normal group (Control), incerebral hemorrhage model group $(\mathrm{ICH})$, the sodium chloride intervention group $(\mathrm{NaCl})$, and rosiglitazone intervention group (RSG). According to a previous study ${ }^{[9]}$, the RSG group was given $3 \mathrm{mg} / \mathrm{Kg}$ of rosiglitazone by gavage 3 days before modeling, $1 \mathrm{~h}$ after modeling, and then on a daily basis until the animal is killed.

\section{Establishment of an ICH model}

$\mathrm{ICH}$ model was performed according to the previously described approach ${ }^{[10]}$. After weighing, $10 \%$ chloral hydrate $350 \mathrm{mg} / \mathrm{kg}$ was intraperitoneally injected for anesthesia. The rats were placed on a stereotactic locator, and their limbs were fixed. After disinfecting the surgical area with iodophor for 2 times, the scalp was cut along the sagittal line, and the sagittal line in the anterior fontanelle was exposed; the anterior fontanelle was $1 \mathrm{~mm}$ in front and $3.5 \mathrm{~mm}$ in the right side of the midline. A round hole with a diameter of $1.5 \mathrm{~mm}$ was then drilled with the skull drill to reach the dura surface. After sensing the breakthrough, the drilling was immediately stopped to prevent hurting the brain tissue. 
For the ICH group and RSG group: the tail artery was disinfected, and $1 \mathrm{ml}$ of tail artery blood was extracted and transferred to an EP tube containing anticoagulant. Then $50 \mathrm{uL}$ of non-clotting blood was extracted with a microsyringe and vertically injected along the borehole for about $6 \mathrm{~mm}$, followed by a slow and uniform injection of $10 \mathrm{uL}(1 \mathrm{~min})$, a pause of $1 \mathrm{~min}$, and additional injection of $40 \mathrm{uL}(4 \mathrm{~min})$, followed by a slow exit after $2 \mathrm{~min}$ of needle retention. In the $\mathrm{NaCl}$ group, $50 \mathrm{uL}$ sodium chloride injection was extracted with a microsyringe (needle diameter of $0.6 \mathrm{~mm}$ ) and vertically injected along the borehole for about $6 \mathrm{~mm}$, followed by a slow and uniform injection of $10 \mathrm{uL}(1 \mathrm{~min}$ ), a pause of $1 \mathrm{~min}$, then another injection of $40 \mathrm{uL}$ (about $4 \mathrm{~min}$ ), followed by a slow and uniform injection of $2 \mathrm{~min}$, followed by a slow exit. In the Control group, only the same part of the model group was given a round hole with the same size drilled with a cranial needle, and the puncture needle was slowly withdrawn after $2 \mathrm{~min}$ of retention [11]

The needle hole was filled with bone wax; the wound was sutured and then disinfected with iodine, after which a sterile dressing was applied. The cerebral hemorrhage model was examined using a Longa scoring method ${ }^{[8]}$; a score greater than 0 indicated a successfully established model.

\section{HE staining}

Brain tissue was examined according to Standard Operating Procedure (SOP) procedure. Briefly, samples were fixed, dehydrated, embedded, sectioned (3 $\mu$ m), and stained with eosin. Finally, digital trinocular cameras microscopic camera system was used for slice image acquisition.

\section{Immunohistochemistry}

Brain sections were incubated with the following primary antibodies: CD16 rabbit polyclonal antibody, 1:100 (concentration), CD206 mouse monoclonal antibody, 1:100 (concentration), biotin secondary antibody: goat anti-rabbit/rat working fluid. DAB color development: brownish yellow substances in cytoplasm or intercytoplasm are CD16 and CD206 positive products. The Image-Pro Plus 6.0 Image analysis system was used to determine the optical density of the entire Image collected (integrated optical density, IOD) and Area (Area), the positive cells, and calculate the average optical density (Mean density, MD).

\section{Western blot}

Rat brain tissue blocks were weighed and cut into pieces. The tissue protein extraction reagent added at a ratio of 1:10 ( $\mathrm{g} / \mathrm{mL})$ was used for ice bath homogenate and ultrasonic crushing. After incubation on ice for $20 \mathrm{~min}$, the cells were centrifuged at 10000rpm for $20 \mathrm{~min}$ to collect the supernatant. (2)SDSPAGE gluing and electrophoresis. (3)Transfer film. (4) Immune response: one-step rapid WB kit (Kang century rat CW2030, rabbit CW2029) was added to the blocking solution for 20 minutes. During this period, the 10x lotion in the one-step test kit was diluted into $1 \times$ lotion with distilled water. The secondary antibody HRP was placed into a $4 \mathrm{~mL}$ centrifuge tube, then the primary antibody and the secondary antibody were mixed, incubated at room temperature for $5 \mathrm{~min}$, and the antibody diluent was added in the one-step method. The closed PVDF membrane was washed once with the bleaching solution and incubated with antibodies. (5)ECL chemiluminescence and exposure development

(6)The film was scanned, and gel-Pro Analyzer4 image analysis software was used to measure the gray value of each band for quantitative analysis

\section{Statistical analysis}

SPSS 25.0 software was used for Statistical analysis. Measurement data were shown as means \pm standard deviation. Analysis of variance was used for the mean comparison between groups, and the Tukey-Kramer method was used for the homology comparison within groups. $a=0.05$ was the test criterion. A Pvalue $<0.05$ was considered as statistically significant.

\section{Results}

\section{Neurological function score after ICH modeling}

The neurological function score of the rats after ICH modeling is shown in Table 1-1 and Figure 1-1:1. There was no statistical difference in the Longa score between the Control group and the $\mathrm{NaCl}$ group $(\mathrm{P}>0.05)$. The Longa scores of the ICH group and the RSG group were significantly higher than those of the Control group and the $\mathrm{NaCl}$ group $(\mathrm{P}<0.05)$. Pairwise comparison showed that the Longa score at $6 \mathrm{~h}$ to $48 \mathrm{~h}$ in the ICH group was higher than that of the RSG group $(P<0.05)$, while the difference between the ICH group and the RSG group was not statistically significant at $72 \mathrm{~h}(P>0.05)$.

Table 1-1. Neurological function scores of each group at different time points 


\begin{tabular}{|c|c|c|c|}
\hline Group & $6 \mathrm{~h}$ & $24 \mathrm{~h}$ & $48 \mathrm{~h}$ \\
\hline Control & $0 \pm 0^{*} \#$ & $0 \pm 0^{* \#}$ & $0 \pm 0^{* \#}$ \\
\hline ICH & $3.00 \pm 1.10^{\& \# \$}$ & $2.33 \pm 0.82^{\& \# \$}$ & $2.17 \pm 0.98^{\& \# \$}$ \\
\hline RSG & $1.5 \pm 1.51^{\& * \$}$ & $1.17 \pm 0.98^{\& *}$ & $1.00 \pm 0.89^{\& *}$ \\
\hline $\mathrm{NaCl}$ & $0 \pm 0^{\# *}$ & $0.33 \pm 0.82^{\# *}$ & $0.33 \pm 0.82 *$ \\
\hline$F$ & 14.143 & 11.280 & 9.018 \\
\hline$P$ & 0.001 & 0.001 & 0.001 \\
\hline
\end{tabular}

\section{Changes of ICH brain anatomy in rats}

From the rat brain tissue's coronal profile, the needle entry channel can be clearly seen, forming a hematoma on the right side of the brain (Figure 1-1).

\section{Morphological characteristics of HE staining in brain tissue of ICH group rats}

At $6 \mathrm{~h}$ after modeling, the pia meninges were intact, the cells in each layer were closely arranged, the nerve cells were slightly edematous, the cytoplasm and cc $24 \mathrm{~h}$, the pia meninges were intact, and a small area of hemorrhagic lesions was found at the edge of the medulla area. A small number of neurons were denat 1B). At 48h, the pia meninges were intact, and a large flaky hemorrhage lesion was seen at the edge of the medulla area, and some neurons around the hemor $\mathrm{ICH}$ group was intact at $72 \mathrm{~h}$. Diffuse hemorrhagic foci were observed in the medulla area, and more inflammatory cells were observed in the foci, mainly lymp was obvious, the interstitial space was widened, a large number of neurons were denaturated or necrotized. No other obvious pathological changes were obse

\section{Immunohistochemical results}

\section{Expression of CD16 in the surrounding tissues of a cerebral hemorrhage in each group}

Average optical density was used to reflect the expression of CD16 in the surrounding tissues of a cerebral hemorrhage in each group. CD16 was expressed in hours after modeling $(P<0.05)$. Except for the Control group $(P>0.05)$, the CD16 expression at different time points in the ICH group, RSG group, and NaCl gre $\mathrm{ICH}$ time. Pairwise comparison showed that the expression level of CD 16 in the RSG group was higher than that in the ICH group at $6 \mathrm{~h}$, and the difference was

\section{Expression of CD206 in the surrounding tissues of a cerebral hemornhage in each group}

Average optical density was used to reflect the expression of CD206 in the surrounding tissues of a cerebral hemorrhage in each group. The CD206 was expre at $24 \mathrm{~h}$ and $48 \mathrm{~h}(\mathrm{P}<0.05)$. There was no difference in $\mathrm{CD} 206$ expression between the Control group and $\mathrm{NaCl}$ group at different time points $(\mathrm{P}>0.05)$. Howev group at different time points $(P<0.05)$. Pairwise comparison showed that the expression level of CD206 in the RSG group was higher than that in the ICH grc

\section{WB results}

\section{Expression of CD16 protein in the surrounding tissues of a cerebral hemormage in each group}

WB was used to detect the expression of CD16 protein in the surrounding tissues of each group. CD16 was expressed in all groups, and there were statisticalls significant difference in CD16 expression at different time points between the Control and $\mathrm{NaCl}$ groups $(P>0.05)$. Contrary, significant differences were found comparison showed that the expression of CD16 in the RSG group was lower than that in the ICH group at $6 \mathrm{~h}(\mathrm{P}<0.05)($ Figure 2-4 and Figure 2-5).

\section{Expression of CD206 protein in the surrounding tissues of cerebral hemorrhage in each group}

CD206 protein was expressed in all groups, with statistically significant differences between the groups at different time points $(P<0.05)$. There was no signif points $(P>0.05)$. Pairwise comparison showed that the EXPRESSION level of CD206 in the RSG group was higher than that in the ICH group at $6 \mathrm{~h}$ and $24 \mathrm{~h}(\mathrm{P}$

\section{Immunohistochemistry and WB were used to detect the expression of CD16 and CD206 in ICH and RSG groups}

As shown in Figure 2-8, CD16 peaked at $6 \mathrm{~h}$ in both the ICH and RSG groups and then gradually decreased within 24-72h. CD206 reached its peak at 24h.

As shown in Figure 2-9, both detection methods showed that the expression of CD16 in the RSG group at different time points was increased compared with t increased compared with that in the ICH group. After the rosiglitazone intervention, the expression of CD16 and CD206 in the RSG group was still higher than 1

\section{Discussion}

Despite the overall increase in preclinical studies, the pathophysiology of ICH remains unclear, and there is still no effective treatment for ICH ${ }^{[12]}$. Microglia,

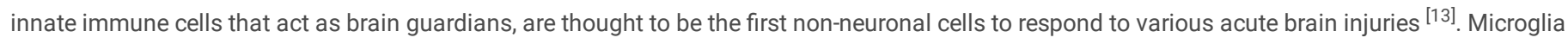
polarization mediates a series of inflammatory cascades, which is the main cause of secondary brain injury after intracerebral hemorrhage. In the early ICH stage, microglia are activated by blood components and polarized into M1-type phenotypes (CD16 markers). M1-microglia increase inflammation, exacerbating brain damage in the early stage after $\mathrm{ICH}$. With the decrease of the inflammatory response, microglia polarized into M2 phenotype (CD206 marker), promoting hemolytic tumor absorption and neurological function recovery ${ }^{[14]}$. Regulating the change of microglia phenotype can accelerate the absorption of hematoma and edema and contribute to tissue repair and remodeling, improve the integrity of white matter, brain repair, and functional recovery. Promoting the polarization of M2-type microglia with neuroprotective effects may be a potential therapeutic strategy for ICH treatment ${ }^{[15]}$.

In this study, we first used the Longa score to assess the neurological behaviors of the mice before and after modeling. The score was slightly higher in the $\mathrm{NaCl}$ group compared to the Control group, although the difference was not significant. This was mainly due to normal saline's compression effect, which could act as the hematoma's physical compression effect. These data suggested that the hematoma's physical compression, if not in a critical position, has little impact on post-ICH survival and further neurological dysfunction.

Blood components contained in autologous blood can stimulate a series of inflammatory reactions in local brain tissue at the puncture site, which is basically similar to the evolution of human cerebral hemorrhage. As expected, the ICH group and the RSG group's Longa scores were significantly higher than those of the Control group and the $\mathrm{NaCl}$ group $(\mathrm{P}<0.05)$. Cerebral hemorrhage was further confirmed by histopathology (the hematoma cavity was distributed in the vicinity of the basal ganglia and compressed the surrounding tissues; HE staining showed that the cell edema of ICH group was obvious), suggesting that the 
model was well established. Moreover, the pairwise comparison showed that the Longa score at $6 \mathrm{~h}$ to $48 \mathrm{~h}$ in the ICH group was higher than that of the RSG group $(P<0.05)$, but not at $72 h(P>0.05)$.

Rosiglitazone, a potent agonists of PPAR y, can coordinate microglia from the pro-inflammatory M1 phenotype to the anti-inflammatory M2 phenotype, thereby inhibiting inflammation and tissue repair ${ }^{[17]}$. Other studies have confirmed that rosiglitazone may further promote the M1-M2 phenotype transfer through activation of the LKB1-AMPK signaling pathway ${ }^{[18]}$. Studies have also found that rosiglitazone can effectively reduce the early inflammatory gene expression and neuron injury, and improve the clearance of hematoma in the ICH model of mice, thus highlighting its neuroprotective effect on the secondary brain injury after cerebral hemorrhage ${ }^{[19]}$. In this study, immunohistochemistry and Western blot were used to further verify that rosiglitazone may be induced by microglia to M2 polarization and have a role in neural function protection. The expression of CD16 and CD206 was found in all groups. The expression levels of CD16 and CD206 in the Control group and NaCl group did not change with time. The expression level of CD16 in the ICH group and RSG group was significantly higher than that in the Control group. It also reached the highest point at 6 hours, after which it gradually decreased with time.

Immunohistochemical expression of CD16 and CD206 was always higher in the RSG group than in the ICH group at $6 \mathrm{~h}$ and $24 \mathrm{~h}$, and the increased amplitude of CD206 was higher than that of CD16 in the ICH group, which was further confirmed by Western Blot. These data suggested that rosiglitazone can enhance the expression of CD16, and especially CD206. The presence of M2-type microglia further speeds up eliminating hematoma and strengthening the nerve protective effect, and in turn, reduces the secondary injury after intracerebral hemorrhage.

In summary, this study showed that the PPAR agonist rosiglitazone could enhance the expression of M1, and especially M2 microglia around the ICH hematoma, which protects the nerves and reduces the secondary injury after intracerebral hemorrhage. However, whether RSG directly stimulates microglia to

directly polarize into M2-type microglia or further transforms into M2-type microglia by reducing m1-type microglia polarization is still unclear, and needs to be further explored.

\section{References}

[1] E P, I B, E N, et al. Microglial HO-1 induction by curcumin provides antioxidant, antineuroinflammatory, and glioprotective effects[J]. Molecular nutrition \& food research, 2015, 59(9): 1690-700.

[2] Y T, W L. Differential Roles of M1 and M2 Microglia in Neurodegenerative Diseases[J]. Molecular neurobiology, 2016, 53(2): 1181-1194.

[3] Xiong X Y, Liu L, Yang Q W. Functions and mechanisms of microglia/macrophages in neuroinflammation and neurogenesis after stroke[J]. Prog Neurobiol, 2016, 142: 23-44.

[4] Wen L, You W, Wang H, et al. Polarization of Microglia to the M2 Phenotype in a Peroxisome Proliferator-Activated Receptor Gamma-Dependent Manner Attenuates Axonal Injury Induced by Traumatic Brain Injury in Mice[J]. J Neurotrauma, 2018, 35(19): 2330-2340.

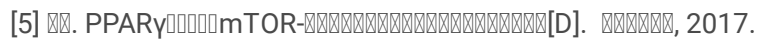

[6] Wu G, Wu J, Jiao Y, et al. Rosiglitazone infusion therapy following minimally invasive surgery for intracerebral hemorrhage evacuation decreases matrix metalloproteinase-9 and blood-brain barrier disruption in rabbits[J]. BMC Neurol, 2015, 15: 37.

[7] Mu Q, Wang L, Hang H, et al. Rosiglitazone pretreatment influences thrombin-induced phagocytosis by rat microglia via activating PPARgamma and CD36[J]. Neurosci Lett, 2017, 651: 159-164.

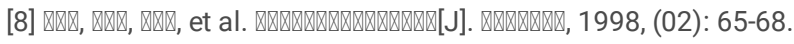

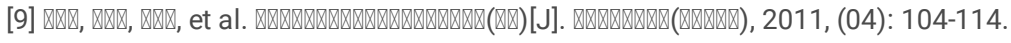

[10] Gy Y, Al B, TI C, et al. Experimental intracerebral hemorrhage: relationship between brain edema, blood flow, and blood-brain barrier permeability in rats[J]. Journal of neurosurgery, 1994, 81(1): 93-102.

[11] Cc K, Mc N, Cl H. Brain trauma elicits non-canonical macrophage activation states[J]. Journal of neuroinflammation, $2016,13(1): 117$.

[12] Dasari R, Zhi W, Bonsack F, et al. A Combined Proteomics and Bioinformatics Approach Reveals Novel Signaling Pathways and Molecular Targets After Intracerebral Hemorrhage[J]. J Mol Neurosci, 2020.

[13] Lan X, Han X, Li Q, et al. Modulators of microglial activation and polarization after intracerebral haemorrhage[J]. Nat Rev Neurol, 2017, $13(7)$ : 420-433.

[14] Zhang Z, Zhang Z, Lu H, et al. Microglial Polarization and Inflammatory Mediators After Intracerebral Hemorrhage[J]. Mol Neurobiol, 2017, 54(3): 18741886.

[15] J L, K N, G B, et al. Post-stroke treatment with argon attenuated brain injury, reduced brain inflammation and enhanced M2 microglia/macrophage polarization: a randomized controlled animal study[J]. Critical care (London, England), 2019, 23(1): 198.

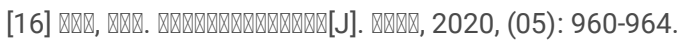

[17] Li Y, Zhu Z Y, Lu B W, et al. Rosiglitazone ameliorates tissue plasminogen activator-induced brain hemorrhage after stroke[J]. CNS Neurosci Ther, 2019, 25(12): 1343-1352.

Page $5 / 9$ 
[18] Ji J, Xue T F, Guo X D, et al. Antagonizing peroxisome proliferator-activated receptor gamma facilitates M1-to-M2 shift of microglia by enhancing autophagy via the LKB1-AMPK signaling pathway[J]. Aging Cell, 2018, 17(4): e12774.

[19] Han C, Xia X, Jiao S, et al. Tripartite motif containing protein 37 involves in thrombin stimulated BV-2 microglial cell apoptosis and interleukin 1 beta release[J]. Biochem Biophys Res Commun, 2019, 516(4): 1252-1257.

\section{Figures}

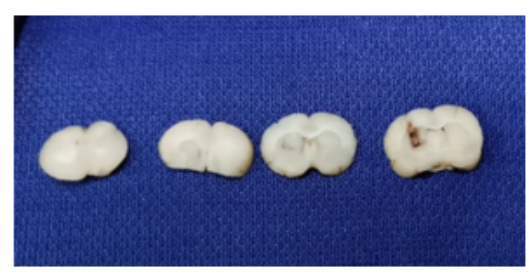

\section{Figure 1}

Anatomical changes in the posterior ICH brain of rats.
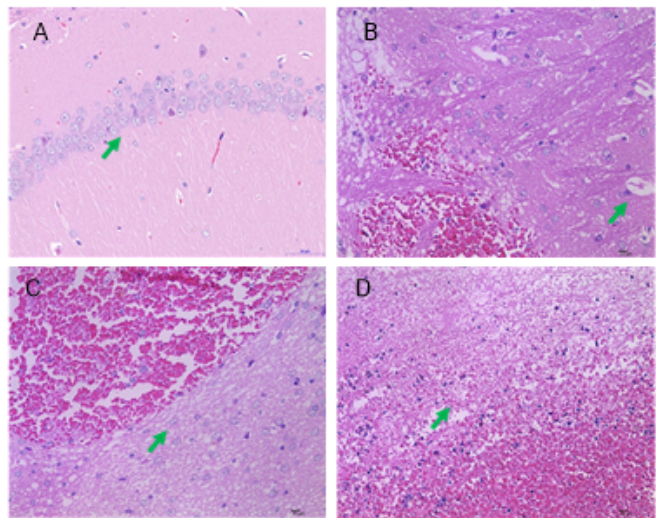

\section{Figure 2}

HE staining of rat brain tissues in ICH group at different time points under a light microscope $(\times 400)$. (A) 6 hours; visible pia meningeal structural integrity $(\uparrow)$. (B) 24 hours, visible nucleation ( $\uparrow$ ). (C) 48 hours; visible cytoplasmic loss ( $\uparrow$ ). (D) 48 hours, with neutrophil infiltration ( $\uparrow$ ) visible.
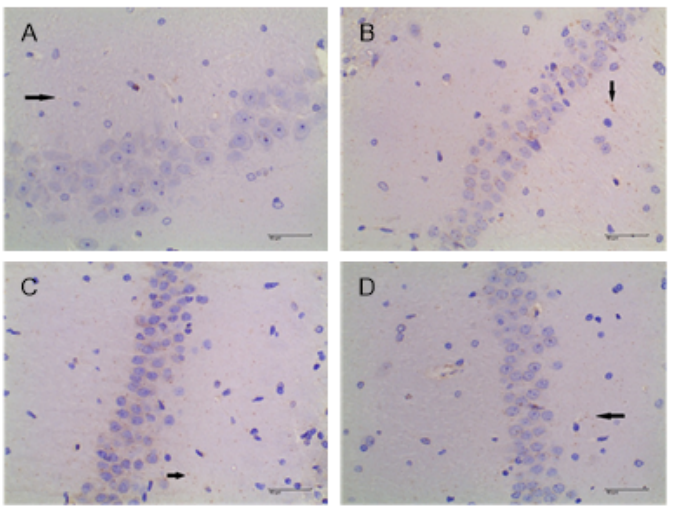

\section{Figure 3}

Expression of CD16 protein around hematoma in each group 6h after modeling (×400). (A) Control group; (B) ICH group; (C) RSG group; (D) NaCl group. Arrows indicate CD16 expression. 

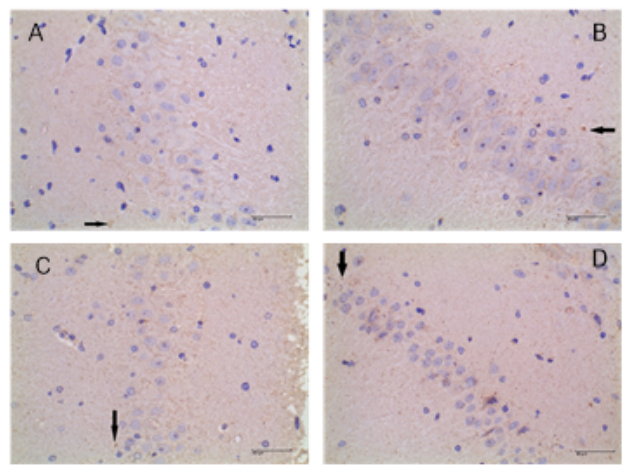

\section{Figure 4}

Expression of CD206 protein around hematoma in each group 24h after modeling (×400). (A) Control group; (B) ICH group; (C) RSG group; (D) NaCl group. The arrow is CD206

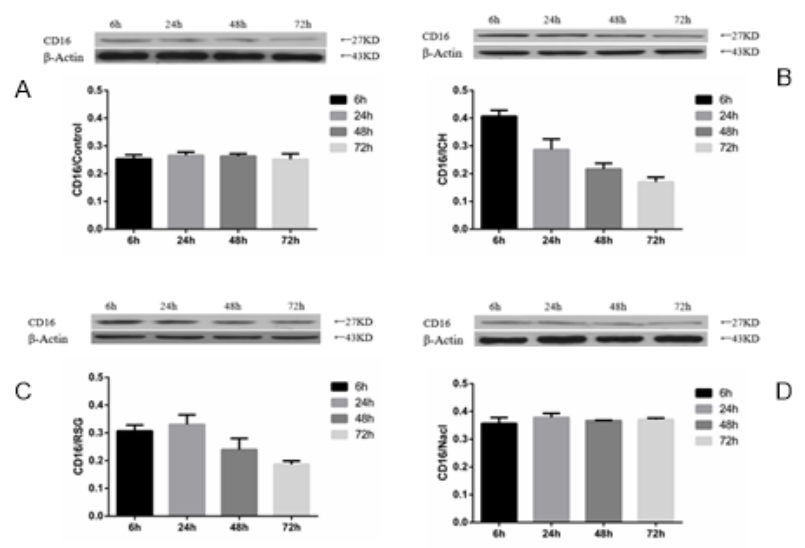

\section{Figure 5}

Expression of CD16 in the same group at different time points. (A) Control group; (B) ICH group; (C) RSG group; (D) NaCl group.
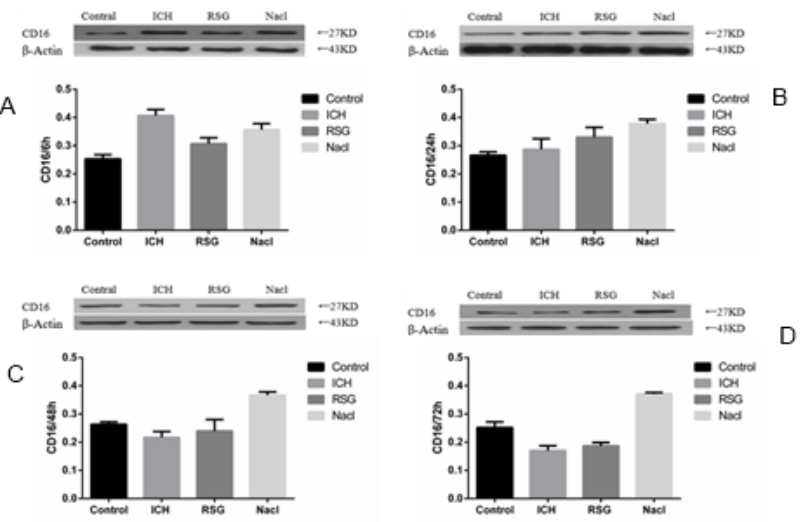

\section{Figure 6}

Expression of CD16 protein in different groups at the same time point. (A) 6h; (B) 24h; (C) 48h; (D) 78h. 

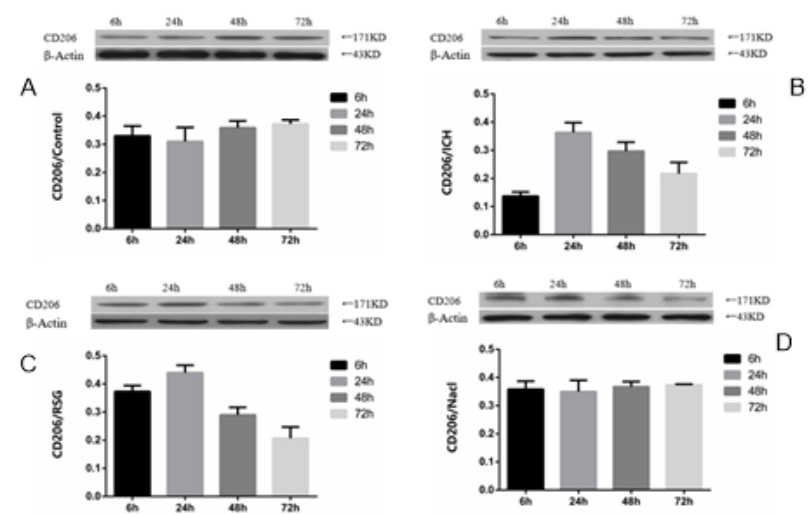

\section{Figure 7}

Expression of CD206 in the same group at different time points. (A) Control group; (B) ICH group; (C) RSG group; (D) NaCl group.

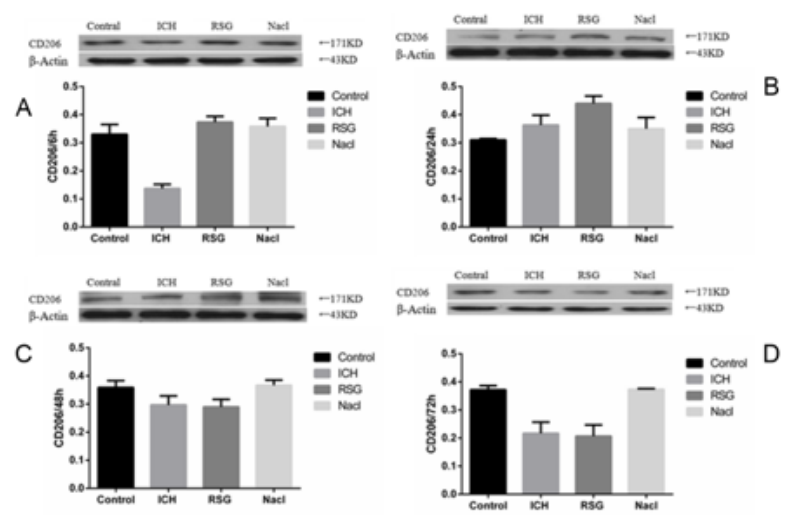

\section{Figure 8}

Expression of CD206 protein in different groups at the same time point. (A) 6h; (B) 24h; (C) 48h; (D) $78 \mathrm{~h}$.

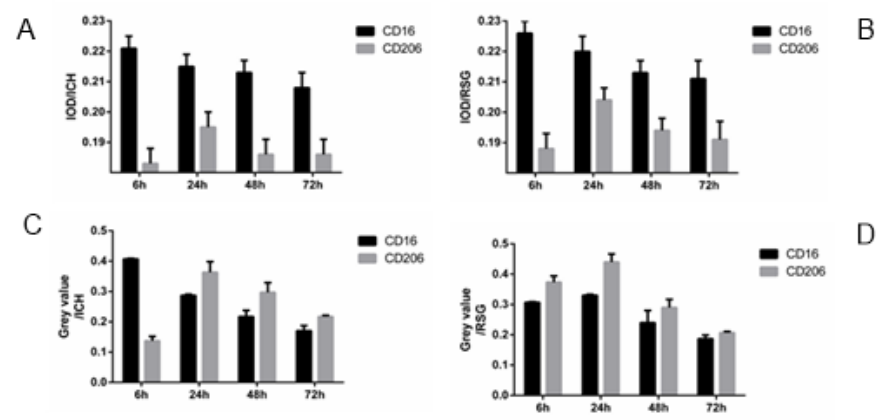

Figure 9

Expression of CD16 and CD206 in ICH group and RSG group at different time points. 


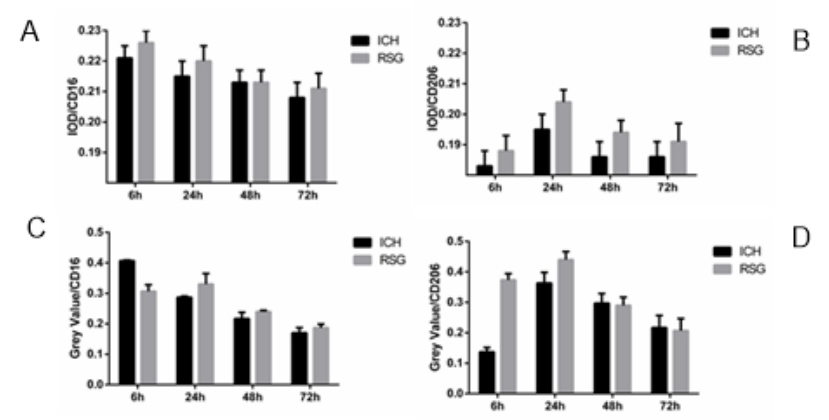

Figure 10

Expression of CD16 and CD206 in the ICH group and the RSG group at different time points 